Part of Journal of Research of the National Bureau of Standards, Volume 23, October 1939

\title{
RADIUM EXPOSURE METER
}

\author{
By Leon F. Curtiss
}

\section{ABSTRACT}

The meter for radium exposure described in this article is a modification of a portable Geiger-Müller counter unit with a range of sensitivity that is suitable for indicating when the tolerance dosage for gamma-ray exposure has been exceeded.

A milliammeter indicates the actual exposure in roentgens per day, and a light and buzzer are energized when the exposure exceeds the equivalent of 0.1 roentgen per day.

This alarm arrangement is particularly important in handling radium, since it reveals at once when the safe limits of exposure have been exceeded. It has been found of help in ascertaining the effectiveness of lead screening arrangements and storage safes. Where large quantities of radium are in constant use, unsafe conditions may frequently pass unnoticed for a considerable time in the absence of some provision for their detection. This device is of great help in training technicians to follow safety procedures by warning them immediately of unsafe conditions, as far as general exposure is concerned.

The device works from a single alternating-current outlet and will operate continuously. It may be transferred readily to any location where a test of exposure is desired.

\section{CONTENTS}

I. Introduction

II. Description of circuit

III. The counter

IV. Adjustment and use of the instrument

\section{INTRODUCTION}

In all institutions where large quantities of radium are handled, it is difficult to be certain that the general exposure to gamma-radiation is within the tolerance dosage of 0.1 roentgen per day. It is particularly important to be certain of this at locations more or less continuously occupied by technicians. Although charts and tables are available to indicate safe screening at various distances, when radium is being constantly moved about, these safe conditions may unconsciously be exceeded. It is even possible that unsafe conditions once created may persist for a considerable time, unless daily routine inspections are made to ascertain the actual conditions. Such inspections are difficult to make and, since they consume considerable time, are frequently neglected. A device which would reveal at a glance the actual intensity at a given location in terms of roentgens per day would do much to prevent even temporary overexposure at a given station. 
A modification of a portable Geiger-Müller counter equipment which is suitable for determining exposure conditions has been developed by the writer and is described below. This device is arranged to give the exposure in terms of roentgens per day and gives an alarm when the intensity exceeds the tolerance dosage. By its use it is possible to protect workers at their regular stations from a general exposure to gamma-radiation in excess of that regarded as safe. ${ }^{1}$

Furthermore, it is an excellent aid in showing technicians immediately when unsafe conditions have arisen from improper storage of radium and is a great aid in creating respect for safety in general. It should be pointed out that a device of this kind does not give warning of temporary local overexposure. Since prevention of local overexposure is a matter of careful routine under suitably arranged and controlled conditions, and only occurs during actual manipulation of the radium, it can be avoided through careful instruction and supervision. General overexposure is more insidious and can affect, without their knowledge, many individuals who are not actually handling radium. It is felt, therefore, that the device described below should help to bring such conditions to light.

\section{DESCRIPTION OF CIRCUIT}

For general convenience, it was decided to use a circuit which could be energized from a single alternating-current outlet. To take care of fluctuations in line voltage, a self-regulating $B$ supply was used to insure that the plate voltages of the amplifier remained sensibly constant. A modification of the Neher-Harper circuit was used so that relatively high counting rates for the Geiger-Müller counter could be used. The amplified and leveled pulses were rectified and fed into a condenser. The indicating meter reads the voltage on the condenser. This arrangement is a slight modification of the circuit used in equipment designed and built by the writer for the United States Navy. ${ }^{2}$

The details of the circuit are shown in figure 1, which is a schematic drawing of the wiring and parts. The circuit can be arranged in other ways to make it more compact and reduce the number of tubes and other parts. The one shown is that of the experimental model which has been assembled and tested. It is used as a basis for explanation here, since it reveals more directly the functions of the various parts.

The counter, $G-M$, is connected to a 57 tube, I, to form a NeherHarper circuit so that high counting rates may be used. The plate of this tube is connected to the bigh voltage for the counter through the resistor, $R_{1}$, and to the wire of the counter in the usual way. The pulses from the first stage are leveled in amplitude by the second 57 tube, II, and are fed into it through condenser $C_{1}$. The pulses are then fed through $C_{2}$ to the 27 tube, III, where they are rectified and charge condenser $C_{3}$. The final tube, IV, is connected in a vacuumtube voltmeter circuit to read, by means of the meter, $M$, the voltage on condenser $C_{3}$. This condenser is provided with a resistance leak to ground so that for a constant rate of charging condenser $C_{3}$ comes to a steady voltage.

1 At the present time, a general exposure of 0.1 roentgen per day is regarded as safe. See Handbook H 23 of National Bureau of Standards, on Radium Protection.

2 L. F. Curtiss, J. Research NBS 23, 137 (1937) RP1223. 
The plate voltage for the vacuum-tube voltmeter circuit is supplied from a tap on one of the voltage dividers, $V D$, through resistors $R_{12}$, $R_{13}, R_{14}$ and $R_{3}$. A relay, $Z$, is connected between the midpoint of $R_{13}$ and $R_{14}$ and the plate end of resistor $R_{12}$. The values of these resistors are chosen so that the relay, $Z$, is closed when the plate current in tube IV drops to a definite value. This point is determined from the calibration of the instrument, as described below, and corresponds to a gamma-ray intensity of 0.1 roentgen per day. The lower part of figure 1 is a diagram of the power supply for the amplifier and counter. As shown, the voltage stabilizer composed of the circuits containing

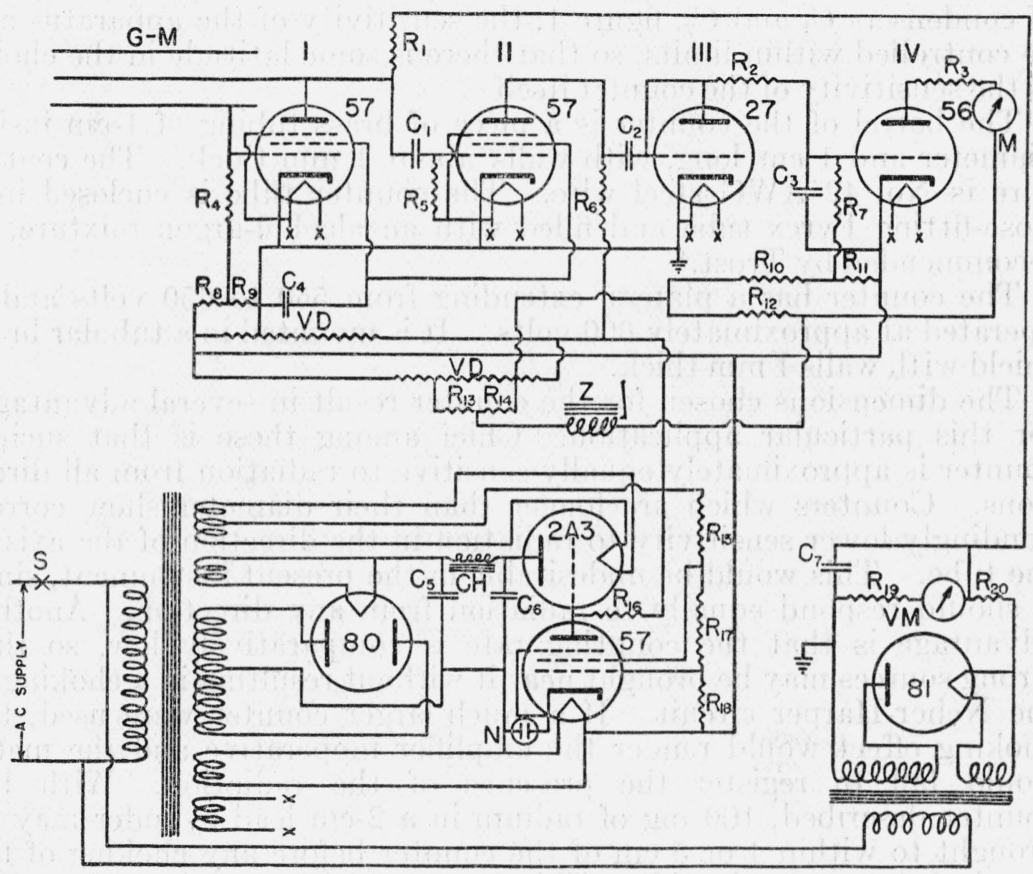

Figure 1.-Wiring diagram of exposure meter.

$R_{1}, R_{5}=5$ megohms; $R_{2}, R_{4}=20$ megohms; $R_{3}, R_{8}, R_{8}, R_{13}=5,000$ ohms; $R_{6}, R_{16}=0.5$ megohms; $R_{7}=4$ megohms; $R_{11}, R_{14}=2,000$ ohms; $R_{10}=0.25$ megohms; $R_{13}=1,000$ ohms; $R_{15}=10,000$ ohms; $R_{17}=27,000$ ohms; $N=H$ neon lamp; $C H=12 \mathrm{~m} h$ ehoke; $R_{18}=11,000$ ohms; $R_{19}=1$ megohm; $R_{20}=0.1$ megohm; $C_{1}=0.0001 f ;$ $C_{3}=0.0002 f ; C_{3}, C_{4}, C_{5}, C_{6}=8 f ; C_{7}=4 f ; G-M=$ tube counter; $V D=$ voltage dividers; $Z=$ relay.

the $2 \mathrm{~A} 3$ and 57 vacuum tubes supplies only the plate voltage for the amplifier. The high voltage from the counter is supplied by a separate transformer and rectifier. A better arrangement would be to take the counter voltage also from the stabilized rectifier. This would eliminate a number of the parts shown and reduce the weight of the instrument. Stabilized high voltage for the counter is not required for counters having a reasonably good plateau.

The circuit can be condensed also by using a single tube having dual electrodes to provide a tetrode and rectifier in the same tube to replace tubes II and III in the amplifier. Such a step is necessary only when space is at a premium, as in portable instruments. 


\section{THE COUNTER}

The intensities to be indicated by the instrument under discussion are in the region of 0.1 roentgen per day, which is a relatively high intensity to measure with a Geiger-Müller counter. It is therefore necessary to use a small counter to obtain the counting rate required, which is of the order of several thousand per minute. This high rate is needed to smooth out statistical fluctuations and give a steady indication on the meter of the instrument for a constant intensity.

By trial, it was found that a counter of the size shown in figure 2 could be used to give a suitable counting rate. By adjusting the size of condensers $C_{1}$ and $C_{2}$, figure 1, the sensitivity of the apparatus can be controlled within limits, so that there is some latitude in the choice of the sensitivity of the counter itself.

The barrel of the counter is a piece of brass tubing of $1-\mathrm{cm}$ inside diameter and $1 \mathrm{~cm}$ long, with walls about $1 \mathrm{~mm}$ thick. The central wire is No. 42 AWG steel wire. The counter tube is enclosed in a close-fitting Pyrex tube and filled with an alcohol-argon mixture, as recommended by Trost. ${ }^{3}$

The counter has a plateau extending from 550 to 650 volts and is operated at approximately 600 volts. It is mounted in a tubular brass shield with walls $1 \mathrm{~mm}$ thick.

The dimensions chosen for the counter result in several advantages for this particular application. Chief among these is that such a counter is approximately equally sensitive to radiation from all directions. Counters which are longer than their diameter show correspondingly lower sensitivity to radiation in the direction of the axis of the tube. This would be undesirable in the present instrument, since it should respond equally to radiation from any direction. Another advantage is that the counting rate is comparatively low, so that strong sources may be brought near it without resulting in a choking of the Neher-Harper circuit. If a much larger counter were used, the choking effect would render the amplifier inoperative and the meter would fail to register the presence of the radiation. With the counter described, $100 \mathrm{mg}$ of radium in a $2-\mathrm{cm}$ lead cylinder may be brought to within 4 or $5 \mathrm{~cm}$ of the counter before any choking of the circuit becomes noticeable. This is a much greater intensity than such an instrument usually would be required to withstand. Finally, the small size of the completed counter tube makes it easy to mount on the amplifier case, so that it is well protected from damage.

\section{ADJUSTMENT AND USE OF THE INSTRUMENT}

The parts described above were mounted in a metal cabinet approximately $15 \mathrm{in}$. high and 7 in. square. The voltmeter of the highvoltage supply and the milliammeter for the vacuum-tube voltmeter were mounted in the sides for convenient observation.

The assembly is shown in figure 3 . The buzzer and warning light are actuated by the relay, $Z$, shown diagramatically in figure 1 .

To adjust the instrument, a radium preparation of approximately $1 \mathrm{mg}$ was used. Such a source in glass gives roughly 9 roentgens per hour at a distance of $1 \mathrm{~cm}$, or about 63 roentgens per working day of

\footnotetext{
${ }^{3}$ A Trost, Z. Physik. 105, 399 (1937).
} 


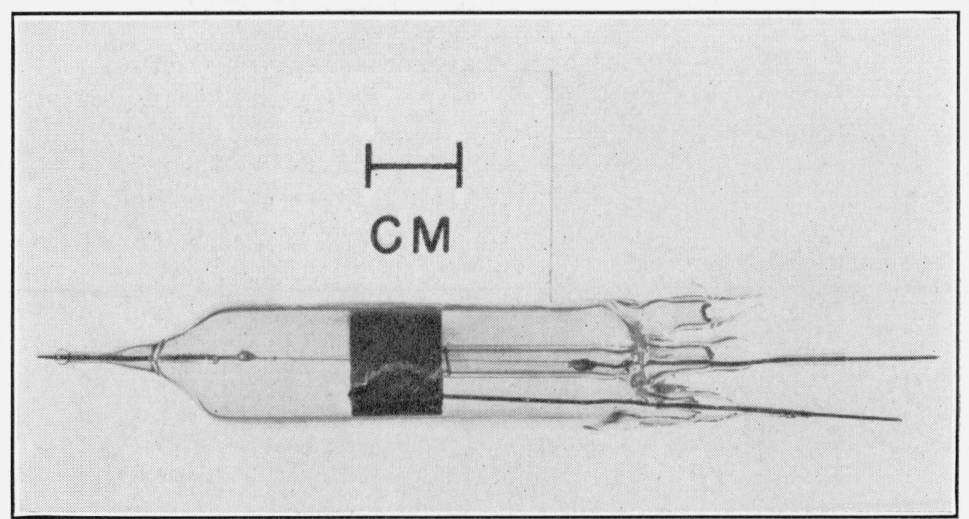

Figure 2.-Tube counter. 


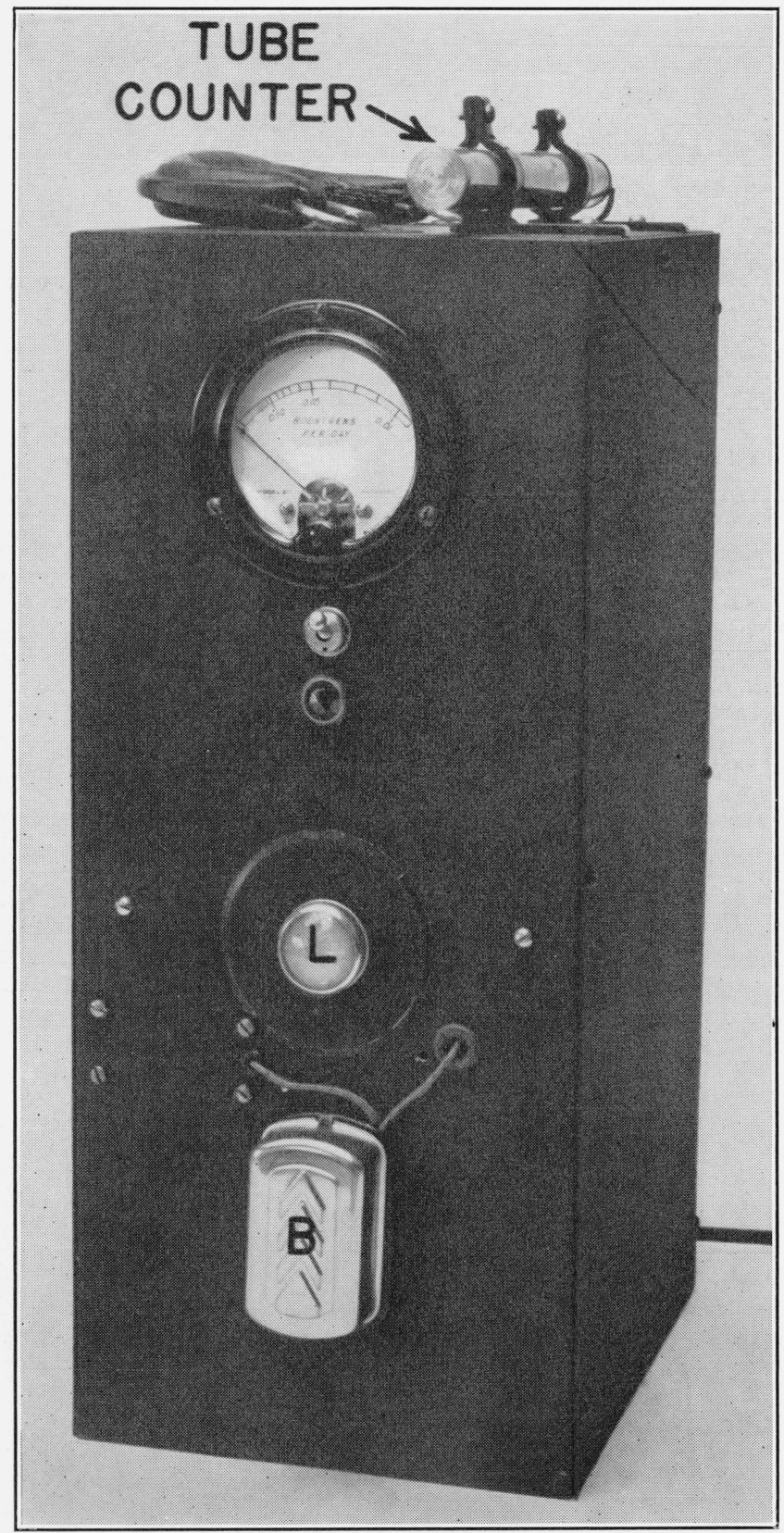

Figure 3.-Completed exposure meter.

The warning light is shown at $L$ and the buzzer at $B$. They are energized when the intensity, as shown on the meter, reaches 0.1 roentgen per day. 
7 hours. Therefore, at a distance of $25 \mathrm{~cm}$ it would give 0.1 roentgen per day, which is the tolerance dose generally recommended.

With the instrument assembled, the radium source was placed $25 \mathrm{~cm}$ from the counter and the sensitivity adjusted by varying the size of the coupling condensers in the amplifier until the steady reading corresponding to 0.1 roentgen per day came just below the linear portion of the operating characteristic of the vacuum-tube voltmeter. The relay circuit was then adjusted, so that the relay would close at this value of the plate current in the vacuum-tube voltmeter.

When the relay closes, a lamp is lighted and a buzzer energized by a separate transformer not shown in the wiring diagram. In this way a direct warning is given whenever the exposure reaches the tolerance level. Approximately 1 minute is required for the instrument to come to a steady reading when the intensity to which it is exposed is suddenly increased from a low value to 0.1 roentgen per day. Therefore, the instrument does not sound a warning when radium is being transported expeditiously through a room or is left

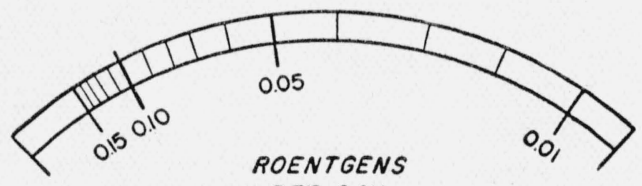

PER OAY

FIGURE 4.-The scale of the indicating meter showing exposures in terms of roentgens per day.

unscreened for a brief interval. Consequently, it responds only to exposures which are unduly prolonged in a way to constitute a real hazard.

By varying the distance between the radium source and the counter, a series of readings were obtained for other intensities of exposure. From these readings a new scale was prepared for the meter of the vacuum-tube voltmeter so that it now reads directly in roentgens per day. A sample scale is shown in figure 4. A glance at this meter reveals the actual exposure at any time with reference to the tolerance dose.

In the form described, this equipment provides a portable unit which operates from an alternating-current outlet. It can be placed near any location which is more or less continually occupied by a worker to determine whether exposure conditions are satisfactory and to give warning when the tolerance dose is exceeded. With slight modification, this unit is also suitable for giving warning on a control board in a central office whenever unsafe conditions arise at various locations in an institution. These modified units could be mounted permanently at locations where possibilities of overexposure exist, with the relay circuit connected to lights on the central control board.

The device described has been tested over a period of months in the radium laboratory at the National Bureau of Standards and found reliable in every respect. By its use several unsatisfactory situations which had existed over a considerable period have been brought to light and corrected. The counters, as made at present, have retained their characteristics over a period of a year and presumably can be relied upon for much longer periods. 
After proper installation such units require very little attention. They have been found to be helpful in revealing situations where overexposures exist and in training technicians to be careful in handling and storing preparations of radium.

The writer acknowledges the aid of L. L. Stockmann and B. W. Brown in constructing the model of the instrument described.

Washington, August 4, 1939. 